\title{
MAPK signaling pathway in development of Alzheimer's disease
}

\author{
N. Muraleva \\ Institute of Cytology and Genetics SB RAS, Novosibirsk, Russia \\ e-mail:Myraleva@bionet.nsc.ru
}

Key words: $\alpha$ B-crystallin, phosphorylation, Alzheimer's disease, OXYS rats

Motivation and Aim: Alzheimer's disease (AD) is an aging-related neurodegenerative disease and accounts for the majority of human dementia. One of the major neuropathological hallmarks of AD is the impairment of proteostasis in the form of deposition of amyloid $\beta$-protein $(A \beta)$ and hyperphosphorylated tau in the brain. The molecular chaperones include $\alpha \mathrm{B}$-crystallin (CryAB) are able to prevent aggregation or misfolding of proteins and enable their correct refolding. Mitogen-activated protein kinase signaling pathway (MAPKsp) is regulate of CryAB activity through its phosphorylation. To investigate a link between alteration of MAPKs pathway and development of sings $\mathrm{AD}$ we compared the gene expression profiles of frontal cortex of OXYS rats and control Wistar rats. Next we compared this result with the CryAB and pS59-CryAB proteins expression and its localization in the brain.

Methods and Algorithms: We used 20-day-old, 5 and 18 mo-old OXYS rats at the presymptomatic, symptomatic, and progressive stage of AD-like pathology, respectively, and Wistar rats as the control $(n=6)$. Analyze of changes in MAPKsp was obtained, using the date of the frontal cortex RNA-seq. CryAB and CryABS59 expressions in brain were indicated by western blot and immunostaining analyzis.

Results: According to KEGG pathway, 27 genes from the MAPKs pathway were changed of which 17 genes were upregulated in cortex of 20 days-old OXYS rats compared to Wistar rats. OXYS rats showed changes in expression of 22 genes, including 15 genes with upregulation, related to MAPKsp at the age of 5 months. The number of genes with altered expression increased to 65 (44 up- and 21 downregulation) by the age of 18 months. Importantly, the mRNA of the MAPKsp activator genes increased with age in OXYS rats. Thus, these results are exactly the same as data of the cortex RNA-seq of the cerebral in humans with AD from open databases. Analysis of protein content in the cortex showed that CryAB level did not differ between OXYS and Wistar rats and did not change with age in the both protein fractions. Phosphorylation of CryaB was absent in detergent - soluble protein fraction of OXYS and Wistar rats. PS59-CryAB was detected only in detergentinsoluble fraction of rats and its level increased with age in the both strain. Importantly, pS59-CryAB level was higher in OXYS rats compared with the Wistar at the age of 5 and 18 mo. This result was confirmed by immunostaining analysis. The pS59-CryAB was localized with $A \beta$ and its co-localization was increased in OXYS rats at 18 mo of age. Conclusion: We showed that the development of the signs of AD in OXYS rat takes place during to alteration of MAPKsp activity in brain cortex. Increase in the level of phosphorylation with age and its co-localization with A $\beta$ may be considered as a compensatory response to the accumulation of aggregates of proteins in OXYS rats. Importantly, mRNA expressions of MAPKsp genes in OXYS rats at the age of 18 mo were same as for humans with AD. These results demonstrate the role of the MAPKsp in the AD suggest that MAPKs could be targeted for novel treatments in the future. Acknowledgements: This study was supported by the RFBR (project No. 18-015-00336). 